\section{Sequential effects in cyclic-interval schedules ${ }^{1}$}

NANCY K. INNIS and J. E. R. STADDON, Duke University, Durham, N.C. 27706

Pigeons were exposed to two cyclic-interval schedules involving a cycle of 12 1-min fixed intervals followed by either 6 2-min intervals or 2 6-min intervals. Response rate was higher during the longer intervals in both cases Comparison between these results and data from a previous experiment with a FI 1 FI 3 schedule suggests that three factors underlie responding to these schedules: the duration of the long intervals, the number of successive long intervals in a cycle (run length), and a factor involving the interaction between interval length and the pigeon's own behavior ("momentum").

Mixed reinforcement schedules consist of two or more alternating schedules in the presence of a single discriminative stimulus (Ferster \& Skinner, 1957; Catania \& Reynolds, 1968). Staddon (1967) examined a mixed fixed-interval fixed-interval schedule in which the intervals alternated in a systematic cyclic fashion. Four cycles, each composed of 12 1-min intervals followed by 4 3-min intervals, were presented in each session. This cyclic-interval schedule produced a cyclic behavioral output: Response rate was higher during the periods when reinforcement density was lower, that is, during the 3-min intervals.

The present experiment examined two related cyclic fixed-interval schedules. \section{SUBJECTS}

Four male White Carneaux pigeons, with previous experience on 1- and 2-min fixed-interval schedules, were maintained at $80 \%$ of their free-feeding weights.

\section{APPARATUS}

A standard Grason-Stadler one-key pigeon chamber was used. The white key light and a houselight provided illumination except during reinforcement (3-sec access to mixed grain), when only a light over the magazine was on. Each recorded peck produced a "feedback" click from a relay mounted in the box. White noise and the noise of the ventilating fan helped mask extraneous sounds. Programming and recording equipment was

Fig. 1. Mean response rate (top) and postreinforcement pause (bottom) for the individual birds for both experimental conditions. Each cycle is the mean of all cycles but the first of a session, averaged over the last 5 days of each condition. in an adjacent room. Data were recorded on digital and printing counters and a cumulative recorder. The postreinforcement pause data were obtained by measuring cumulative records with the aid of a dissecting microscope.

\section{PROCEDURE}

In Condition 1, the birds were run in daily sessions consisting of three cycles (54 reinforcements) of an FI 1 FI 2 cyclic schedule; each cycle involved 121 -min intervals followed by 62 -min intervals. This schedule was in effect for 30 days, after which Condition 2 was instituted. In this condition, the reinforcement schedule was a cyclic FI 1 FI 6 . Each of the 32 daily sessions consisted of four cycles per session, each cycle comprising 121 -min intervals followed by 26 -min intervals ( 56 reinforcements).

\section{RESULTS}

Figure 1 shows response rate and postreinforcement pause during an average cycle for both experimental conditions. Data are averages of the last 5 days of each condition for each of the four pigeons. The first cycle of each session is not included in the average, since behavior during this cycle differed from performance later in the session. Three birds always began sessions with high rates that subsequently declined; the fourth (Bird 32) usually started slowly and increased in rate over the first few intervals.

During the first condition (FI 1 FI 2), three of the four birds responded faster during the 2-min intervals than during the 1-min intervals; Bird 32, on the other hand, responded somewhat more slowly during the 2 -min intervals than during the 1-min intervals. Rate declined somewhat across the six 2-min intervals of each cycle for all four birds. Three of the four birds showed some tendency to longer postreinforcement pauses during 2-min intervals than during 1-min intervals. Pauses during the first 1-min interval of each cycle were about the same as pauses during the preceding 2-min interval, as might be expected on the assumption that the animals were not predicting the interval changes. Thus pauses were longer and response rate lower during the first 1-min interval of each cycle than during later 1-min intervals.

Under the FI 1 FI 6 schedule, all four pigeons responded faster during the long intervals than during the short intervals, al though the difference in rates was less for Bird 32 than for the other birds, as in the first condition. However, response rate during the second 6-min interval of each cycle was always much higher than during the first 6-min interval. Thus, response rate during the first few short intervals of each cycle was higher than during later short intervals. Cumulative records under this condition often showed step-like "scalloping" during the 6-min intervals (cf. Ferster \& Skinner, 1957). These scallops resembled the pattern of responding during the 1-min intervals and often two or three would occur within one 6-min interval. This kind of responding was much less evident during the 2-min intervals of the first condition.

These response rate increases during the long intervals of the FI 1 FI 6 condition reflect changes in postreinforcement pause. Pause during the second 6-min interval of each cycle was much shorter than during the first 6-min interval.

The differences between these two procedures are more easily seen in Fig. 2

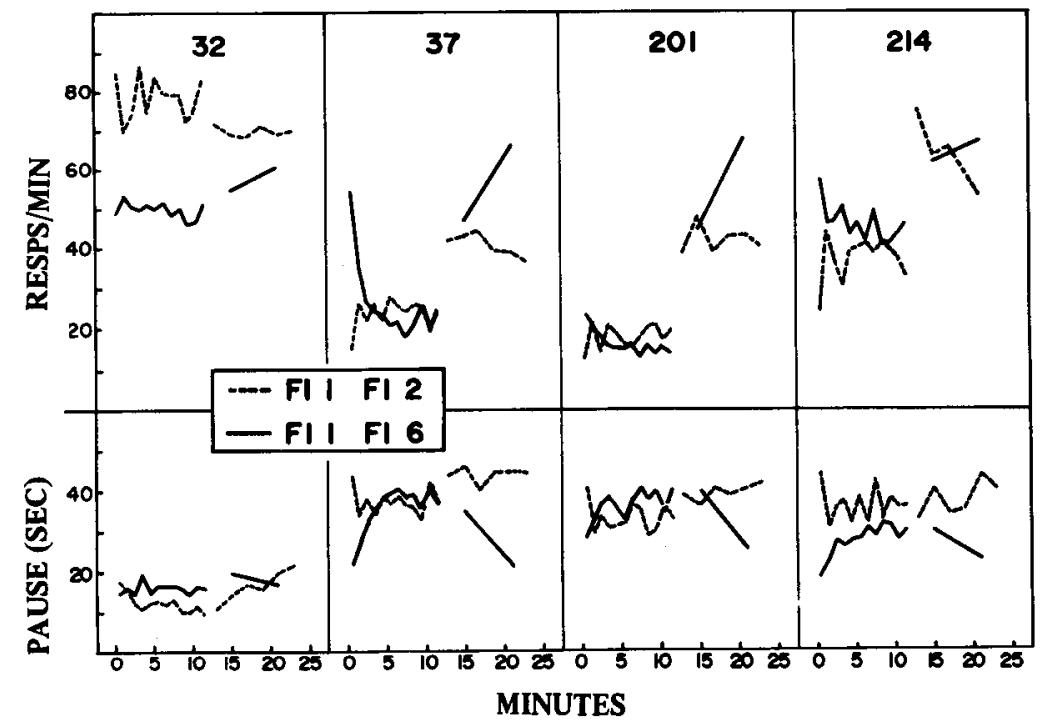




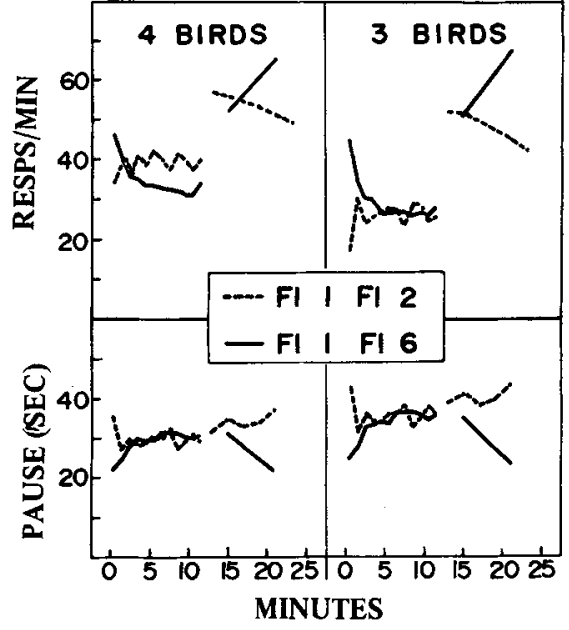

Fig. 2. (Left) An average cycle for the four birds, the mean of the four cycles in Fig. 1. (Right) An average cycle excluding the data for Bird 32.

(left panel), which shows an average cycle for each schedule for the group as a whole. The FI 1 FI 2 procedure showed an approximately constant rate through the short intervals (excepting the first) and a higher, declining rate over the six 2-min intervals. Postreinforcement pause data mirror the rate picture, showing an increasing pause during the long intervals and a long pause during the first short interval of each cycle, with a slightly shorter, constant pause during later short intervals. The picture is almost opposite for the FI 1 FI 6 schedule. Rate declined and pause increased over the short intervals, and rate increased and pause decreased over the long intervals.

The differences between the two procedures are even more clearly shown by the right panel of Fig. 2, which shows an average cycle excluding Bird 32 .

$$
\text { DISCUSSION }
$$

The two schedules of this experiment, and the schedule involving a cycle of 12 1-min intervals followed by four 3-min intervals previously studied (Staddon, 1967, 1969), are all members of a general class of $12 \mathrm{FI} 1, a \mathrm{FI} x$ schedules, where $a x=12$; i.e., mixed schedules with a cycle comprising 121 -min intervals followed by $12 \mathrm{~min}$ of $a$ longer-valued fixed intervals each of duration $x$

The results of this experiment indicate that the typical performance generated by the $12 \mathrm{FI} 1,4 \mathrm{FI} 3$ schedule in the previous experiments is a special case, intermediate between the performances under the two schedules of this experiment. Thus, when the long intervals were FI 2, in this experiment, response rate decreased slightly across the six intervals and pause increased. When they were FI 3 , as in the previous experiments, rate was approximately constant and pause increased only slightly across the four intervals. When the long intervals were FI 6, in this experiment, rate increased sharply (and pause decreased) across the two intervals. In all three schedules, response rate over the long intervals of the cycle was higher than over the short intervals; moreover, this difference was greatest for the FI 1 FI 6 schedule and least for the FI 1 FI 2 schedule.

The differences in responding during the 1-min intervals may be related to the changes in the long intervals by the assumption that the birds were not able to predict the transitions from one interval value to the other with any accuracy. The basis for this assumption, which may not reflect any absolute limitation on the capacities of these animals, is the similarity between postreinforcement pauses during the last short (long) interval of each cycle and the first long (short) interval (cf. Fig. 2). Thus, the decreasing rate and lengthening pause during the six 2-min intervals of the FI 1 FI 2 schedule cycle entailed a longer pause, hence lower rate, during the first short interval of each cycle. However, one or two short intervals sufficed to restore pause and rate to their baseline 1-min values under this schedule. The picture under the FI 1 FI 3 schedule studied previously was quite similar: A small increase in pause during the long intervals entailed a drop in rate during the first short interval. In this case, response rate during the second short interval rebounded slightly and baseline values were not obtained until the third or fourth short interval of the cycle (cf. Staddon, 1967, Fig. 3). Finally, under the FI 1 FI 6 schedule, the sharply decreasing postreinforcement pause across the two 6-min intervals produced a higher rate during the first short interval of each cycle.

Rate continued to decline thereafter, not reaching a baseline value, until after four or more short intervals (cf. Fig, 1).

Three factors seem to be involved in determining the pattern of responding under these cyclic schedules:

(1) The difference in absolute fixed interval duration between long and short intervals; presumably the larger the difference between long and short intervals, the more likely that the birds' behavior will be differentially controlled by long and short intervals. Postreinforcement pause, especially, might be expected to become longer during longer intervals by comparison with short intervals. This outcome was obtained only in the case of the FI 1 FI 2 schedule, however. During the other two schedules, pause either remained the same (FI 1 FI 3 schedule) or actually decreased (FI 1 FI 6 schedule) during the longer intervals, and response rate was higher during the long intervals under all three schedules. This result suggests the involvement of a second factor that acts in opposition to fixed-interval duration.

(2) The number of successive long intervals within each cycle (run length) is such a factor, since all three of the schedules discussed here are characterized by having the product of the number of longer intervals times the length of each interval a constant. Thus, as the duration of the longer fixed-interval increased across these three schedules, the run length necessarily decreased. Since an increase in run length might be expected to facilitate differential control of behavior by the short and long intervals, a decrease should impair it, and thus these two factors-run length and interval duration-may have been acting in opposition to each other in these schedules. Moreover, the decrease in run length from six, in the FI 1 FI 2 schedule, to two, in the FI 1 FI 6 schedule, seems to have outweighed the concomitant increase in duration of the longer interval, since postreinforcement pause was actually less during the second long interval of the latter schedule than during the short intervals. This characteristic of responding under the FI 1 FI 6 schedule, points to a third factor that may be involved here.

(3) A very long fixed interval interpolated in a sequence of short intervals will generally produce, for fairly obvious reasons, a large number of responses and a high rate just preceding eventual reinforcement. The "momentum," to use Sherrington's simile, associated with this kind of behavior may impede subsequent discrimination of fixed-interval duration, despite the large difference between short and long intervals which, in itself, might be expected to aid discriminability. This third factor is suggested by the finding that postreinforcement pause decreased from the first to the second 6-min interval of each cycle during the FI 1 FI 6 condition. Parametric experiments in which run length and absolute interval duration are systematically and independently varied may help decide on the relative contributions of these three factors.

\section{REFERENCES}

CATANIA, A. C., \& REYNOLDS, G. S. A quantitative analysis of the responding maintained by interval schedules of reinforcement. Journal of the Experimental Analysis of Behavior, 1968, 11, 327-383 (Whole Part 2).

FERSTER, C. B., \& SKINNER, B. F. Schedules of reinforcement. New York Appleton-Century-Crofts, 1957.

STADDON, J. E. R. Attention and temporal 
discrimination: Factors controlling responding under a cyclic-interval schedule. Journal of the Experimental Analysis of Behavior, 1967, 10, 349-359.

STADDON, J. E. R. Multiple fixed-interval schedules: Transient contrast and temporal inhibition. Journal of the Experimental Analysis of Behavior, 1969, 12, 583-590.
NOTE

1. Research supported by Grants MH 11525 and MH 14194 from the National Institute of Mental Health and grants from the National Research Council of Canada. Reprints may be obtained from J. E. R. Staddon, Department of Psychology, Duke University, Durham, N.C. 27706.

\section{Effects of neonatal testosterone on the}

\section{acquisition of an active avoidance response in genotypically female rats}

\author{
WILLIAM W. BEATTY and PATRICIA \\ ANN BEATTY, North Dakota State \\ University, Fargo, N. Dak. 58102
}

Female rats received either testosterone or placebo injections at 3 days of age and placebo, ovariectomy plus placebo, ovariectomy plus estrogen, or ovariectomy plus testosterone treatments in adulthood. Females that received testosterone both in infancy and in adulthood exhibited the inferior avoidance performance typical of normal males; the other seven groups behaved like untreated females. Testosterone appears to organize neural mechanisms responsible for sex differences in avoidance behavior, but additional androgenic stimulation is required for the expression of masculine avoidance behavior.

The presence or absence of testosterone during a critical period in development organizes structures in the anterior hypothalamus so that either a tonic (male) or a cyclic (female) pattern of gonadotrophin release results (Barraclough, 1967; Gorski \& Wagner, 1965). Despite many attempts to demonstrate that the capacity to display masculine or feminine sexual behavior also depends on early exposure of the hypothalamus to testosterone, the issue is still unresolved. Although treatments that prevent the maturation of the feminine pattern of gonadotrophin release generally depress feminine sexual behavior (Barraclough \& Gorski, 1962; Harris \& Levine, 1965), they do not necessarily potentiate masculine sexual behavior (Mullins \& Levine, 1968; Whalen, Edwards, Luttge, \& Robertson,
1969). Prolonged exposure to androgens during development produces female rats that display complete masculine copulatory responses (Ward, 1969), but the changes in the size and morphology of the external genitalia produced by this treatment are so pronounced that it is impossible to determine whether the behavioral changes result from hormonal influences on the development of the brain or the phallus (Nadler, 1969).

The study of behaviors where sex differences exist, but where gross differences in genital structure are unlikely to affect performance, provides an opportunity to evaluate the organizational influence of early hormone treatments on neural and other systems, free from the confounding effects of alterations in phallic morphology. Female rats acquire a two-way active avoidance response more rapidly than males and they do not display the marked intersession performance decrement characteristic of males (Levine \& Broadhurst, 1963). ${ }^{1}$ We attempted to determine if these sex differences were related to the presence of testosterone early in development.

\section{METHOD}

Female rats of the Holtzman strain received injections of testosterone propionate $(1.25 \mathrm{mg})$ or an equivalent volume $(.05 \mathrm{cc})$ of sesame oil placebo at 3 days of age. When the rats reached 91 days of age each of these early treatment groups ( $T$ or $P$ ) was divided into four subgroups: ovariectomy plus placebo replacement therapy (Group TOP, $N=8$, and Group POP, $N=10$ ); ovariectomy plus estradiol replacement (Group TOE, $\mathbf{N}=9$, and Group POE, $N=10$ ); ovariectomy plus testosterone replacement (Group TOT, $\mathrm{N}=10$, and Group POT, $\mathrm{N}=11)$; or control operations plus placebo injections (Group TCP, $\mathrm{N}=9$, and Group PCP, $N=11$ ). Replacement therapy began on the 9 th postoperative day; daily subcutaneous injections continued for the remainder of the experiment. Groups POT and TOT received $1 \mathrm{mg}$ per day testosterone propionate; Groups TOE and POE received 1 microgram per day estradiol benzoate; other groups received an equivalent volume $(0.10 \mathrm{cc})$ sesame oil placebo.

Avoidance tests were conducted in two identical shuttleboxes made of aluminum and Plexiglas. These have been described elsewhere (Schwartzbaum, Green, Beatty, \& Thompson, 1967). Seven days after the start of replacement therapy, tests of spontaneous activity and the tendency to escape the CS (offset of light) were conducted in the shuttleboxes. There were no group differences in activity and all animals rapidly adapted to the CS. Avoidance training began on the following day and continued for 3 consecutive days. Each daily session consisted of 50 trials presented on a 60 -sec fixed-interval schedule. The CS preceded the onset of shock by 10 sec and was terminated by an avoidance response (moving to the other side of the box). Otherwise, the CS overlapped presentation of the shock and both CS and US were terminated by an escape response. The shock US was delivered from a high-voltage ac source via a grid scrambler. US intensity was $1.0 \mathrm{~mA}$. RESULTS

As seen in Table 1, testosterone

Table 1

Mean Performance During Avoidance Tests*

\begin{tabular}{lrcc}
\hline Group & N & $\begin{array}{c}\text { Avoidance } \\
\text { Responses }\end{array}$ & $\begin{array}{c}\text { Decrement } \\
\text { Score }\end{array}$ \\
\hline TOT & 10 & 73.5 & 4.50 \\
POT & 11 & 115.2 & 0.09 \\
TCP & 9 & 106.0 & 0.63 \\
PCP & 11 & 113.5 & 0.60 \\
TOP & 8 & 113.3 & 0.88 \\
POP & 10 & 119.1 & 0.60 \\
TOE & 9 & 109.7 & 0.38 \\
POE & 10 & 112.9 & 0.33 \\
Normal & 10 & 98.8 & 3.50 \\
Males & & & \\
Normal & 10 & 115.0 & 0.50 \\
Females & & &
\end{tabular}

- Avoidance responses are three session totals (150 trials). Decrement score equals the number of avoidance responses on the last ten trials of Session 2 minus the number of avoidance responses on the first ten trials of Session 3. Datal from groups of normal males and females of the same age and strain tested under identical conditions, but raised by the supplier are included for comparison. These data were not included in the statistical analyses. 